\section{MS13-05 Structure and dynamics of the polymyxin-resistance-associated response regulator PmrA in complex with the promoter DNA}

Chwan-Deng $\mathrm{Hsiao}^{1}$

1. Institute of Molecular Biology, Academia Sinica

email: hsiao@gate.sinica.edu.tw

PmrA, an OmpR/PhoB family response regulator (RR), manages genes for antibiotic resistance. Phosphorylation of OmpR/PhoB RR induces the formation of a symmetric dimer in the $\mathrm{N}$-terminal receive domain (REC), promoting $2 \mathrm{C}$-terminal DNA-binding domains (DBDs) to recognize the promoter DNA to elicit adaptive responses. Recently, determination of the KdpE-DNA complex structure revealed a unique asymmetric REC-DBD interface that may be necessary for transcription activation. Here, we report the $3.2-\AA$ resolution of the crystal structure of the PmrA-DNA complex, which reveals a similar yet different asymmetric REC-DBD interaction. However, this asymmetric interface is transiently populated in solution, as revealed by relaxation dispersion NMR, and is not crucial in supporting gene expression, as evidenced by the b-galactosidase reporter assay. The interdomain dynamics of the protein, together with the DBD-DBD interface, may help PmrA search for the most suitable conformation for interacting with RNA polymerase holoenzyme to activate downstream gene transcription.

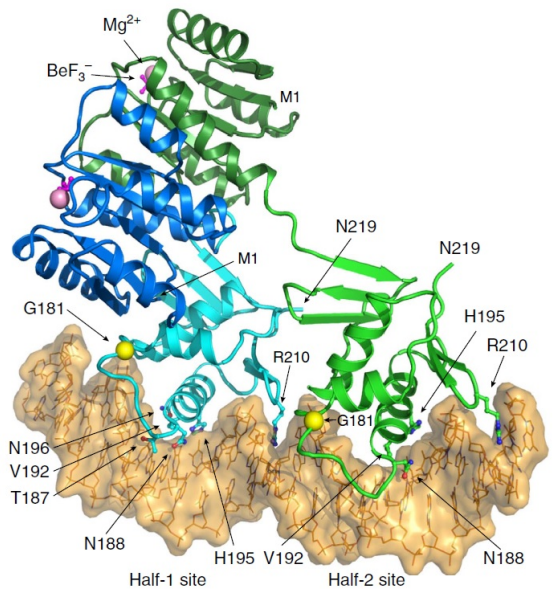

Figure 1. Structure of the $\mathrm{BeF}_{3}^{-}$-activated $\mathrm{PmrA}$ dimer in complex with the promoter DNA. The PmrA molecule that recognizes half-1 site is denoted as PmrA-1 and half-2 site as PmrA-2.

Keywords: Two-component systems, response regulator, crystal structure
MS14 Biomineralogical

crystallography and bioinspired

inorganic materials

Chairs: Wolfgang Schmahl, Fernandez-Diaz Lurdes

MS14-01 Learning from Sea Shells -

Bio-Inspired Approaches Toward

Mesoscale Architectures in Functional Spinel Oxides

Anna S. Schenk ${ }^{1}$, Sabine Eiben ${ }^{2}$, Miriam Goll ${ }^{1}$, Lukas Reith ${ }^{1}$, Elisabeth John ${ }^{1}$, Alex N. Kulak ${ }^{3}$, Fiona C. Meldrum ${ }^{3}$, Christina Wege $^{2}$, Sabine Ludwigs ${ }^{1}$

1. Institute of Polymer Chemistry, University of Stuttgart, Pfaffenwaldring 55, 70569 Stuttgart, Germany

2. Institute of Biomaterials and Biomolecular Systems, University of Stuttgart, Pfaffenwaldring 57, 70569 Stuttgart, Germany

3. School of Chemistry, University of Leeds, Woodhouse Lane, LS2 9JT, Leeds, United Kingdom

email: anna.schenk@ipoc.uni-stuttgart.de

Despite drawing on a limited pool of available elements, nature has developed sophisticated mechanisms to fabricate nanostructured minerals under environmental conditions. Biominerals characteristically represent organic/inorganic composite materials with hierarchical organization spanning multiple length scales, where these complex architectures often lead to astounding properties adapted to suit a specific function. Unsurprisingly, the study of biominerals has therefore inspired a substantial body of research activities, which are aiming to transfer key concepts of biological mineralization, e.g. the use of structure-directing organic matrices and confined reaction environments, into artificial systems. This approach is particularly exciting in view of the growing demand for eco-efficient synthetic routes for the preparation of technologically relevant materials showing inherent functional properties.

We here explore bio-inspired strategies to generate mesoscale architectures in spinel cobalt(II,III) oxide $\left(\mathrm{Co}_{3} \mathrm{O}_{4}\right)$ and related mixed metal spinels, where these compounds represent a highly promising class of materials for a wide range of applications, particularly in the field of heterogeneous catalysis. In this context, the catalytic activity of the functional oxide largely depends on its morphology, nanostructure and surface area. Specifically, we precipitate thermally unstable cobalt hydroxide carbonate precursors under biomimetic conditions in the presence of either synthetic water-soluble polymers with flexible chains or tobacco mosaic viruses (TMV), where the latter represent plant-derived nanoscopic biotemplates with a rigid-rod supramolecular structure. Calcination at relatively moderate temperatures converts the mineral precursors into the functional spinel oxide phase, while retaining the 